\title{
LES EXPOSITIONS D'AUTOMNE
}

\author{
Par M. GuY RIORGES
}

\section{TOIN}

Le 29 Septembre... c cest un peu löt pour nous.

L'Union n'était donc pas convoquée à l'exposition agricole de Lyon, organisée par le P.-L.-M. aveé la collaboration des Faux et Forèts. Vous verrez que nous y étions pourlant, gràce à MM. Raybato et LovbrT.

Ne : łécrivons pas le stand puisqu'une image nous en reste. C'est un fameux coup d'oeil.

M. Altairac, Inspecteur principal à Lyon, avait meublé to bacs d'Anguilles, Perches, Ablettes, Coujons, Chevaines, Brochets, Barbeaux, Truites et larves de Lamproie.

M. Kneitmans, Inspecteur ì Thonou, exposait ja bacs d'alevins de Truites arc-en-ciel et albinos, des 'Truites, Ombres et Ombles chevaliers. Il avait aussi 6 petits bacs de faune et flore aquatique oi les Daphnies semblaient sélectionnées, tant clles étiient grosses. Il paraìt très bon de montrer les bestioles utiles ou nuisibles, les plantes à conserver ou à détruire : cette initiative doit ètre suivie, car elle est aussi instructive qu'amusante et décorative.

M. Joubert, Inspecteur à Nìmes, avait envoyé, en bocaux, Muges, tloses et Esturgeons. Notons que la présentation en bocal et celle de sujets disséqués ou naturalisés, peul ìtre très intéressante ; j'espère toujours que l'Linion réunira un musée de ce genre. Qutre les animaux-types, on y verrait, avec plaisir, des spécimens présentant les déformations les plus curieuses, les traces d'aceidents remarọuables. Qui n'a regretté, un soir de peche, de ne ponvoir conserver une larpe dont la peau, la chair ou l'ossature raconte un drame étommant : Qui nous enseignera l'art de préparer la béte, el qui nous dolia des bocaux destimés à enrichir les collections de l'Union!

M. Joubert avait installé un lastor de Beancaire et deux Myopotames qui ont eu grand el légitime succès. I ce propos, observons que l'exposition de betes à fourrures, daus la salle voisine, tendait à la diffusion de l'Ondatra : or, volis savez combien le prince Cor.ourno-Mansolito s'est repenti d'avoir introduit che\% lui ce lapin-mat d'eau, qui n'a pas frayé avec la Carpe, mais qui a pullulé el causé d'importants ravages. Jeannotlapin troue nos chanssées : que dirons-nous quand nous y verrons fouir l'Ondatra échappé du pare d'un éleveur voisin? Le Chat et la Perchesolejl, ces rastaquouères désastreux dont 11 . It: Conservateur Galloos m'a fait constater de formidables méfaits dans le has cours du Rhône, ne nous suffisent-ils pas .comme leçon? 


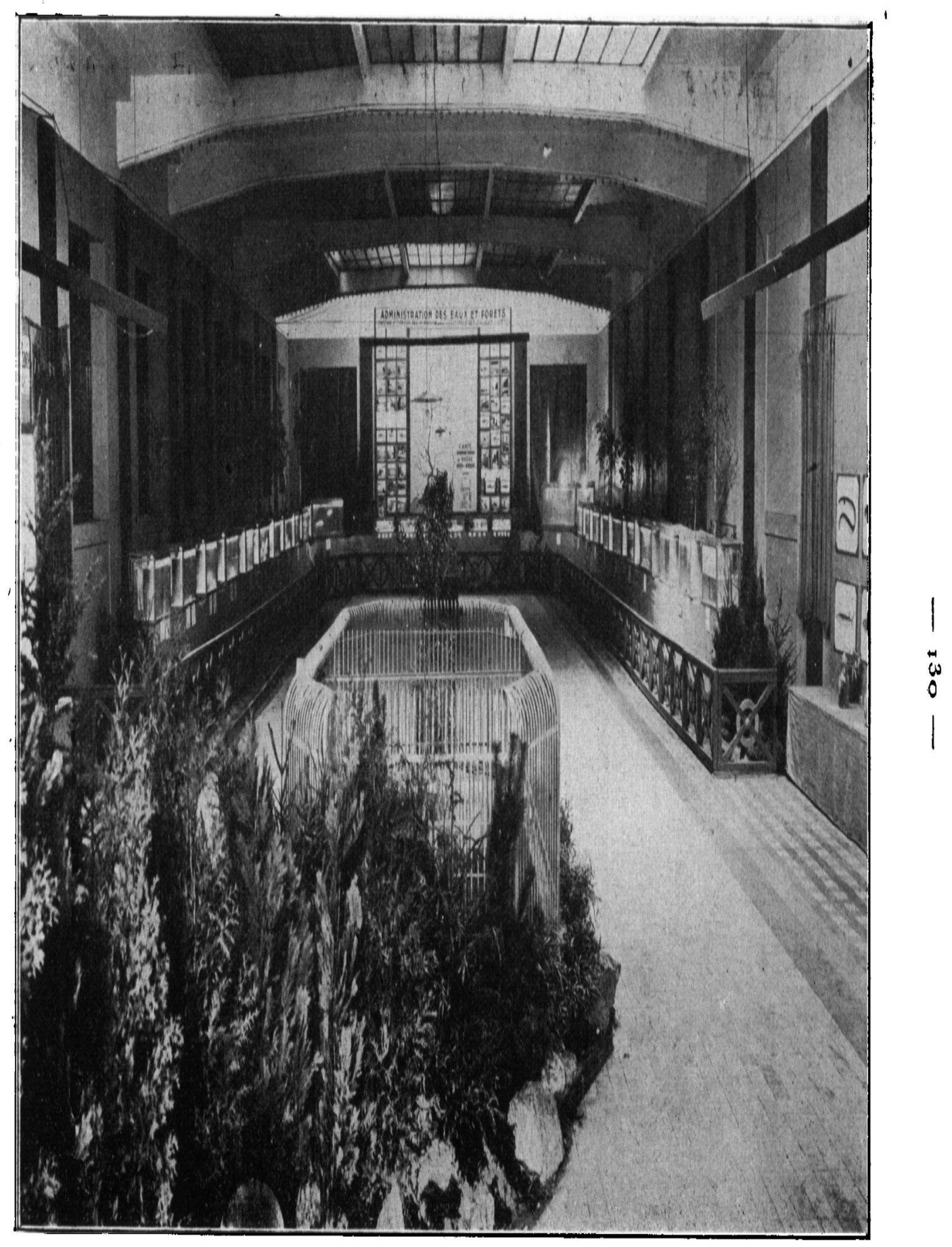

Exposition de Lyon, - Salle de la pisciculletere. 
Revenons à la belle exposition du P.-I.-M., que M. le Professeur Roure ornait des aquarelles saisissantes de M. AxGer, son assistant au Muséum, et où M. le Professeur LÉger avait envové ses collections méthodiques et précieusement documentaires de cartes et de photographies.

La carte piscicole du Khône, due à M. Kreistmanis, nous fait souvenir de l'intérêt qu'aurait une carte générale, comme en ont Varsovie et Berlin.

M. le Conservateur de Valence avait inslallé des appareils d'incubation peuplés qui retenaient l'attention.

Et les étangs? Nous y voilà.

Malgré la saison, M. Rameaux occupait 3 bacs par un contingent très remarquable, quoique évidemment cueilli au hasard du filet : Carpes Wittingau de 3 étés, Tanches de 3 étés, feuilles de Carpes, auves de Tanches, Gardons, Ablettes. Ce producteur sait ce qu'il convient de mettre dans ses étangs pour en tirer une marchandise facilement écoulable et rentable : c'est un guide sûr et courlois.

Le Syndicat du Forez avait envové les types de ses pèches de septembre : 3 cuirs (si nous disions Royales?) nées en 1926, de la à 6 livres ; r écaille Meugniot de 7 livres, r936 (je n'ose plus parler des Wittingau du Forez après la spirituelle lance rompue par le Secrétaire de la rédaction du Bulletin contre le bordeaux de Crimée : mais n'y a-t-il pas de charolaises en Forez? Alors...) Je ne reprocherai que deux choses à ces Carpes : mal à l'aise par la chaleur, elles s'agitaient si bien qu'elles se meurtrissaient ; et le Forez tend, à présent, à faire du 3 élés trop gros. Nous y avons vu une pèche de is.ono livres ne comportant que 2.200 têtes : une moyenne de 2.735 grammes. C'est peut-être de l'art ou du sport, ce n'est plus le rapport maximum. Préférons une autre pêche, de 2 étés celle-là, où l'étang ì donnf́ son plein rendement avec des nourrains de 2 à 3 lives fort réguliers. Le Forez en exposait à Lyon, 3 cuirs, 3 écailles, identiques de poids. Mais c'est là de la Carpe bonne marchande et il eut fallu exposer des nourrains d embouche de 300 ou foo grammes : ce n'est pas plus difficile à obtenir que ceux de 3 livres, et c'est plus utile pour les éleveurs.

De la belle feuille cuir, née a'un rroisement de génileurs Meugniot et Hirsch, en juin 1928 .

Des Tanches 1927 de 200 kmmmes avec leurs atues rg28, car la Tanche laissée au large grossit vite el fraie en gragnant son second été.

Eufin quelques Carpes curituses : moussue ; nordue par le brochet à la lèvre ; ensellée par accident ; evaguérément bossue par arrêt de développement caudal ; bâtarde de poisson rougee.

Il est impossible d'accueillir des Poissons malades dans des bacs à eau communiquante, malgré le courant d'évacuation : ce serait pourtant instructif. Ne pourrait-on faire à leur usage une section spéciale ? 


\section{M耳TZ;}

Le i Octobre, ciétait à Metz qu'il fallait prendre la legon de choses.

Certes, le vaillant Syndicat lorrain n'a pas les moyens dı P.-L.-M., mais il n'en est que plus intéressant d'observer son effort et sa réussite. Quel réconfort, quelle vague d'espérance et de confiance au milieu de ce groupe précis, actif, agissant et que rien ne rebute ! Les difficultés d'organisation vaincues par M. Peurlox l'affirment un chef et un débrouillard. Avec rien, il a réussi à se procurer tout le nécessaire, et au delà. C'est très bien, mais l'union se doit de seconder mieux de tels propagandistes et de mettre de tels organisateurs à mème de mener ses afliaires avec les leurs. Que nous faut-il pour ètre riches: In peu d'argent de première mise. Aurons-nous l'esprit de verser I franc pour en gagner 50 ou 100 ? Qui vivra verra...

Les Lorrains n'ont pas que de la tète ct des nerfs : ils ont de belles Carpes.

Le Lindre expose un couple de géniteurs de 6 étés, type miroir, resté presque pur, au moins quant à la femelle qui est de formes parfaites el pèse : I livres. Le mâle est long, fort en tête : la saison n'a pas permis d'en pêcher un meilleur, mais ils ne doivent pas manquer, si l'on en juge par le lot de feuilles r $9^{2} \delta$ qui présentent toutes les caractéristiques de la perfection.

M. Paté envoie 4 magnifiques 3 étés écailles de 4 livres, à tète remarquablement petite et à dos charnu.

M. Colignos expose une femelle écaille qui eùt peut-ètre emporté le prix d'honneur sur celle du lindre, si l'àge avait été indiqué. On ne peut rien voir de plus beau quant aux formes, ni, si eile a jótés, quant au poids.

Ses feuilles, ses nourrains, cuir et écaille, sont racés à souhait, et ses Tanches vertes sont très bien venues.

Les curiosités ne manquent pas : Carassins, l'erches, Brochels, Inguilles, Goujons, Ablettes et Ecrevisses énormes.

Ce ne serait déjà pas mal. Les Lorrains ont fait pluss, el l'on voit qu'outre l'élevage, ils songent également au matériel, en v rais civilisés.

Un camion équipé à l'oxygène contint pendant plusieurs jours un chargement de Carpes de 6 à i 2 liv res qui démontrent l'excellence de ce transport. Une locomotive silme au loin : on se prend à songer. Ceci tuera-t-il cela? M. Peupiox, fui n'est pas un songe-creux, ne s'arrète pas à cette vision lointaine ou proche. I côté du canion, il a monté un bac de transport en wagon alimenté, non plus a l'oxygèue, mais par une pompe très ingénieuse qui paraît hien ouvrir une voie nouvelle et heureuse. Les Carpes y grouillent, pleines, de vie.

Bottes Arraud ; bottes Grinewaid ; propulseur à main "I a queue de poisson " ; outillage Weber ; propulseur Vrenmest ; filets Hecrol ; canols Feyel ; vêtements Dreyfuss ; filets Cocntie; pièges Gmoskry ; barque 
Benser ; diffuseur de 1 Oxhydrique française ; emballages Kollex ; nasses Bernardx ; pièges Galme ; matériel de campement NoIrel ; et la faucardeuse Eurêka! N'est-ce pas une exposition type?

J'oubliais les bocaux à poissons curicux et la décoration florale ravissante, fournie par M. Naver, directeur des promenades.

Certes, le Comité de la Foire a fourni au Syndicat le stand et les installations, et il en faut féljciter $M$. HocọuAhd et M. Rıbière : mais on voit que nos amis lorrains ont su fáire utile et beau à travers toutes les autres exigences d'une exposition. Dieu sait s'il y en a ! M. le Conservateur Allotre a pu voir ce que sait faire un bon Syndicat : l'administration a raison de nous aider.

Le $G^{3 l}$ DE Mondaincount peut être fier de son pays et je pardonne à mon excellent ami, M. DF Cnevigss, la pointe narquoise de son sourire lorsqu'il me demande des nouvelles de mes Carpes. Je me souviens d'avoir vu les siennes à Paris et ce que je vois à Met\% me rend modeste. On peut égaler les Lorrains, mais quant à les dépasser... Ces Carpes représentent évidemment une moyenne, puisque les pèches ne sont pas commencées, et que l'on n'a pu rien choisir. Eh bien, c'est une solide moyenne !

Autre bonne initiative : le déjeuner, menu de Poisson d'étang. Je souhaitais cela depuis longtemps : à Metz, on l'a réalisé.

Et encore la distribution du matériel commandé en commun : M. PEUpion est un réalisateur, voilà le mot. Ajoutez qu'il l'est sans rudesse, avec bonté et que le Syndicat a offert de cotiser jusqu'à cent sous l'hectare pour exécuter le plan de l'Union. Concluez.

\section{$*^{*} *$}

Ces expositions de Lyon, de Met\%, de Limoges, et celle de Paris qui s'annonce pleine de fruits, sont un pas dans la voie de la propagande et une occasion de liaison entre producteurs, et même producteurs et marchands. Connaissons-nous plus et mieux, et nous ferons connaître nos produits, qui le méritent.

Que tous les producteurs actifs se rencontrent, qu'ils annoncent aux hésitants la bonne nouvelle : il y a chez nous les éléments de l'action utile. Il y a des initiatives, des réalisations : voyez la Lorraine, la Touraine, le Limousin, la Franche-Comté, les Dombes, le Forez, etc. Stagnerons-nous encore, quand un petit effort commun nous affranchirait de mainte entrave?

Impossible ! La timidité n'a pas le droit de se déguiser en prudence. Que risquons-nous, en effet : 2o sous l'hectare deux ou trois ans de suite. Ce n'est pas cela qui arrêtera la marche de 300 producteurs résolus. Seulement, le résultat devant profiter à tous, ces 3 on braves ne doivent pas payer pour les 500 autres.

Au fait, messieurs, dites votre avis.

Sur quels points estimez->ous que doive porter notre effort? 
A quels problèmes cherchez-vous d'ahord une solution? Définissez les buts.

Quels moyens voyez-vous d'arriver à ces buts?

Dans une association comme la nôtre, le pire ennemi de l'homme d'action, c'est celui qui n'a pas d'avis, qui s'abstient. Or, qui peut s'abstenir lorsqu'il s'agit de sauver son bien, d'obtenir le prix de son travail?

Dressez la liste de vos espoirs nécessaires.

Etablissez le plan qui doit les réaliser.

Des maîtres ouvriers pour ce faire, je crois qu'il y en a.

\section{LA MÉVENTE DU POISSON \\ Ses Causes et ses Remedes}

\section{Par M. Michel LHÉRITIER}

Comme il était facile de le prévoir, les petites améliorations apportées à nos procédés d'aquiculture et, particulièrement, l'adoption de plus en plus fréquente des pêches annuelles, ont eu pour résultat immédiat de jeter chaque hiver, sur le marché, une quantité de Poisson plus considérable que celle habituelle, qui semblait devoir suffire aux besoins des revendeurs et de leur clientèle. De plus, les conditions économiques présentes ayant modifié la mentalité de nos concitoyens, tels, qui considéraient leurs étangs comme des non-valeurs, ont élé amenés à les estimer comme toutes les autres parties de leurs domaines et à leur demander des ressources aujourd'hui fort intéressantes.

Cependant, dès avant la guerre, et spécialement lors de la Mission piscicole de r9I7 et durant le premier Congrès de l'étang et de l'élevage de la Carpe, en Mars 1918 , je me suis efforcé d'attirer l'attention de nos collègues sur la nécessité de s'assurer des débouchés rémunérateurs pour leur récolte présente, avant nnème d'accroître leur production ; je leur ai exposé les avantages du système que je préconisais, mais, hélas! sans plus de succès que Cassandre prédisant les malheurs de Troie.

Plus tard, j'ai tenté, aussi vainement, de montrer le danger qu'il y avait à négliger le marché intérieur pour l'exportation, prévoyant qu'un jour, assez prochain, cette ressource unique viendrait à manquer. Quoique commerçant moi-même avec l'Angleterre, j'ai, plusieurs fois, demandé au Ministère de l'Agriculture l'interdiction de l'exportation dans le but d'amener les carpiculteurs à rechercher des débouchés régionaux et à contribuer, de la sorte, au ravitaillement national qui fait ou qui doit faire l'objet des préoccupations youvernementales.

Un tolle général a accueilli mes propositions, et plusieurs collègues, gens d'esprit cependant, ont affirmé qu'une telle mesure serait la mort certaine de la carpiculture française. 